\section{Applying the use of novel biomarkers for neuroendocrine tumors in the clinic: where are we now?}

\author{
Kosmas Daskalakis ${ }^{1}$, Olov Norlén ${ }^{1}$, Per Hellman ${ }^{1}$ \& Peter Stålberg *,1 \\ ${ }^{1}$ Department of Surgical Sciences, Endocrine Surgical Unit, Uppsala University Hospital, 75185 Uppsala, Sweden \\ *Author for correspondence: peter.stalberg@surgsci.uu.se
}

\section{"The most important future task in NET management is the development of multianalyte markers"}

First draft submitted: 17 April 2017; Accepted for publication: 26 April 2017; Published online:

12 February 2019

Keywords: biomarkers $\bullet$ neuroendocrine tumors $\bullet$ prognosis $\bullet$ targeted therapy

Neuroendocrine tumors (NETs) arise from the diffuse neuroendocrine system and demonstrate a great diversity in their clinical course with unpredictable and variable outcomes hidden in an overall favorable survival rate, and thus a substantial time to detect changes in disease status. This variability in prognosis combined with delayed diagnosis of NETs often restricts treatment effectiveness. The absence of adequately sensitive and specific biomarkers obviates the need for novel markers that could be used for early diagnosis, prognostication and real-time monitoring of disease progression and treatment response [1].

The general limitation of currently used markers is that they are secretory monoanalyte biomarkers, which lack sensitivity, specificity and predictive ability. Chromogranin A (CgA) is a general marker for NETs, which can also be found elevated in various inflammatory conditions, such as inflammatory bowel disease and rheumatoid arthritis, as well as essential hypertension, chronic heart failure, impaired renal function, pregnancy, steroid treatment or glucocorticoid excess, liver disease, hyperparathyroidism, chronic atrophic gastritis and finally treatment with proton-pump inhibitors (PPI) [1,2]. Moreover, CgA is elevated in other non-neuroendocrine neoplastic tumors, such as pancreatic adenocarcinoma, prostate carcinoma and small-cell lung carcinoma. Additionally, there is a lack of standardization of concentrations between different laboratories and even within commercial tests for CgA and other monoanalyte biomarkers, so that the results may exhibit wide variations being difficult to interpret [2].

Thus, CgA is a nonspecific (10-35\% specificity) and moderately sensitive marker (60-90\% sensitivity) [2]. Nevertheless, it has been proven to be an independent prognostic factor for NETs, associated to tumor burden, recurrence and treatment response [3]. As it is correlated with tumor burden, $\mathrm{CgA}$ tends to be higher in metastatic disease, thus its efficacy in detection of earlier stages of the disease is rather questionable. However, it is more frequently elevated in well-differentiated tumors compared with poorly differentiated ones, and treatment with somatostatin analogs (SSA) seems to reduce the correlation between CgA and tumor burden [3].

Pancreastatin is a polypeptide product of $\mathrm{CgA}$, which has been investigated as a candidate biomarker with clinical significance [4]. This marker has been reported to have greater sensitivity and sensitivity than its precursor CgA, and it is not affected by PPI treatment. However, when compared with CgA, pancreastatin does not correlate with tumor burden and aggressiveness. Moreover, pancreastatin levels are found elevated in patients with diabetes or hyperparathyroidism, and can also be affected by certain medications [4].

Neuron-specific enolase has also been investigated and found to be a potential marker for NETs, mostly for poorly differentiated ones; however, it is not used in clinical practice as it cannot differentiate between different subtypes of NETs and it is generally outperformed by CgA [3].

The 5-HIAA is the breakdown product of serotonin; it is measured in urine and found elevated if the tumor burden is high enough in patients with metastatic small intestine (SI)-NETs [5]. Additionally, there are several medications, including somatostatin analogs or types of food, and malabsorptive conditions that interfere with
Future $\because$ Medicine 
urine 5-HIAA levels. In SI-NETs, 5-HIAA levels demonstrate a high specificity over 90\%, but a rather low sensitivity around $60-70 \%$ with some correlation to overall survival in patients with metastatic disease [6,7].

Monoanalyte markers in serum for specific types of functional gastroenteropancreatic (GEP-NETs) are currently utilized in clinical practice and are considered effective indicators of tumoral activity. These secretory biomarkers, namely insulin and proinsulin for insulinoma, gastrin and progastrin for gastrinoma, glucagon for glucagonoma and vasoactive intestinal peptide (VIP) for VIPoma, address only a subset of patients with NETs, approximately $3-5 \%$ of all NETs $[8]$.

A number of monoanalyte markers, such as tachykinins neurokinin A, pancreatic polypeptide, $\mathrm{N}$-terminal probrain natriuretic peptide, CTGF and progastrin-releasing peptide, have also been investigated and generally demonstrated disappointing metrics for diagnosis, prognostication and monitoring of NET disease [8,9].

There are no specific markers to date for bronchopulmonary NETs, even if these tumors express CgA, serotonin, neuron-specific enolase and gastrin-releasing peptide [10]. The currently used markers for GEP-NETs are inadequate for bronchopulmonary NETs, both in terms of diagnosis and follow-up [11].

Circulating tumor cells (CTCs) are a relative new biomarker for neoplasia, based on the measurement of cellular expression of the epithelial cell adhesion marker [12]. The role of CTC has been investigated in colorectal, breast and prostate cancers with promising results as a prognostic marker of progression-free and overall survival. However, in patients with GEP-NETs, only a minority of cases, up to $43 \%$ for SI-NETs, had detectable CTCs with some correlation to tumor burden in the liver but no correlation to grade and prognosis. Additionally, there was no correlation observed between CTC levels in patients with NETs and treatment response [12,13].

The role of miRNAs as potential biomarkers has also been investigated. The miRNA profiles for GEP-NETs in tumor tissue are dysregulated and distinguishable among NETs of different origin [14,15]. For example, miR-103 and miR-107 are upregulated in pancreatic NETs, and miR-21 is associated with higher grade and metastatic disease [15]. miR-1290 downregulation in serum can distinguish pancreatic NET from adenocarcinomas [14]. However, there is a lack of data on the expression of miRNAs in serum and additionally a lack of standardization and implementation of normalization methods, making the use of miRNAs in clinical practice a challenging future task.

A number of novel biomarkers are currently targeted toward treatment selection and monitoring but most require tumor tissue biopsies. Ki-67 is being widely used in NETs as a proliferative marker, but with a significant inter- and intraobserver variability as well as a lack of uniformity throughout the tumor with highest hotspots defining the tumor grade. No correlation between Ki-67 and treatment response of patients with NETs to chemotherapy has been reported [16]. Oncotype DX and MammaPrint are novel, biopsy-based biomarker assays that predict tumor behavior for breast and colon carcinomas, respectively. Similarly for NETs, somatostatin receptor immunohistochemistry and imaging are being used in order to predict response to treatment with somatostatin analogs and peptide receptor radionuclide therapy [17].

Multianalyte tests may improve diagnostic sensitivity and specificity by exploring additional biomarkers that address monoanalyte marker deficits. Multianalyte assays with algorithmic analyses correlate and normalize multiple sets of variables and are currently used in a broad spectrum of diseases, both malignant and nonmalignant. For example, FibroSure is used for the diagnosis of hepatitis $\mathrm{C}$, and it is measured in patient blood, replacing liver biopsy. Additionally, multiple gene tests have been reported as screening tools in patients with ovarian cancer and chronic lymphocytic leukemia.

Using nucleic acid proximity-based methods, namely proximity ligation assay and its further refinement, proximity extension assay, panels of serum biomarkers have been tested in SI-NET disease [18]. Novel markers identified were TFF3 and Midkine with a diagnostic ability in early disease stages and additionally DcR3, a marker for liver metastases. In terms of prognosis, high levels of DcR3 and TFF3 were linked to shorter overall survival [18]. These new markers may provide a basis for a new set of multianalytes, which remain to be further validated and studied in respect to their predictive ability.

In the light of contemporary translational research on NETs and the development of novel targeted drugs, pretreatment markers, which will be exploited as predictive factors of response, are needed. Mutational status in the PIK3CA/Akt/mTOR pathway has failed to predict response to everolimus treatment, probably due to errors related to epigenetic changes, thus protein and/or mRNA expression markers in this pathway could be studied in order to select patients for treatment with mTOR inhibitors. Regarding other targeted novel therapies, IL-8, sVEGFR-3 and SDF- $1 \alpha$ were recently identified as predictors of response to tyrosine kinase inhibitor sunitinib in a Phase II study [19]. Moreover, O6-methylguanidine-DNA methyltransferase expression is currently evaluated as a predictor of response to temozolomide treatment. 
A gene biomarker called the 'NETest' has been developed recently, comprising a validated circulating 'fingerprint' of NETs detected by gene transcript PCR analysis. This NET multianalyte assay with algorithmic analysis biomarker has been shown to be a more robust method compared with commonly used monoanalytes, with a reported sensitivity of $98 \%$ and a specificity of $97 \%$ [20]. The gene transcript PCR analysis is standardized and not affected by PPI and different types of food. Moreover, it has demonstrated promising results regarding its ability to provide therapeutically relevant information in terms of monitoring disease progression and treatment response, with the latter being the best added value of this test compared with monoanalytes. Apart from this, there may be an additional role of the NETest in clinical practice in the assessment of the completeness of locoregional surgery as well as the efficacy of resective surgery/ablative methods of metastatic liver disease. The utility of NETest in non-GEP-NETs and/or mixed epithelial-NETs remains to be further elucidated and is currently being evaluated in clinical trials.

\section{Conclusion}

The most important future task in NET management is the development of multianalyte markers, which, combined with novel functional imaging, will better address the NET complexity, provide multidimensional information alongside the development of novel targeted therapies in the era of translational research, replace minimally invasive procedures and facilitate the implementation of personalized management of patients with NETs.

Financial \& competing interests disclosure

The authors have no relevant affiliations or financial involvement with any organization or entity with a financial interest in or financial conflict with the subject matter or materials discussed in the manuscript. This includes employment, consultancies, honoraria, stock ownership or options, expert testimony, grants or patents received or pending, or royalties.

No writing assistance was utilized in the production of this manuscript.

\section{Open access}

This work is licensed under the Attribution-NonCommercial-NoDerivatives 4.0 Unported License. To view a copy of this license, visit http://creativecommons.org/licenses/by-nc-nd/4.0/

\section{References}

1. Modlin IM, Oberg K, Taylor A, Drozdov I, Bodei L, Kidd M. Neuroendocrine tumor biomarkers: current status and perspectives. Neuroendocrinology 100(4), 265-277 (2014).

2. Modlin IM, Gustafsson BI, Moss SF, Pavel M, Tsolakis AV, Kidd M. Chromogranin A - biological function and clinical utility in neuro endocrine tumor disease. Ann. Surg. Oncol. 17(9), 2427-2443 (2010).

3. Yao JC, Pavel M, Phan AT et al. Chromogranin A and neuron-specific enolase as prognostic markers in patients with advanced pNET treated with everolimus. J. Clin. Endocrinol. Metab. 96(12), 3741-3749 (2011).

4. Rustagi S, Warner RR, Divino CM. Serum pancreastatin: the next predictive neuroendocrine tumor marker. J. Surg. Oncol. 108(2), 126-128 (2013).

5. Kema IP, de Vries EG, Muskiet FA. Measurement of 5-HIAA in urine. Ann. Clin. Biochem. 32(Pt 1), 102-104 (1995).

6. Meijer WG, Kema IP, Volmer M, Willemse PH, de Vries EG. Discriminating capacity of indole markers in the diagnosis of carcinoid tumors. Clin. Chem. 46(10), 1588-1596 (2000).

7. Formica V, Wotherspoon A, Cunningham D et al. The prognostic role of WHO classification, urinary 5-hydroxyindoleacetic acid and liver function tests in metastatic neuroendocrine carcinomas of the gastroenteropancreatic tract. Br. J. Cancer 96(8), 1178-1182 (2007).

8. Eriksson B, Oberg K, Stridsberg M. Tumor markers in neuroendocrine tumors. Digestion 62(Suppl. 1), 33-38 (2000).

9. Korse CM, Taal BG, Bonfrer JM, Vincent A, van Velthuysen ML, Baas P. An elevated progastrin-releasing peptide level in patients with well-differentiated neuroendocrine tumours indicates a primary tumour in the lung and predicts a shorter survival. Ann. Oncol. 22(12), 2625-2630 (2011).

10. Righi L, Volante M, Rapa I, Scagliotti GV, Papotti M. Neuro-endocrine tumours of the lung. A review of relevant pathological and molecular data. Virchows Arch. 451(Suppl. 1), S51-S59 (2007).

11. Oberg K, Hellman P, Ferolla P, Papotti M, Group EGW. Neuroendocrine bronchial and thymic tumors: ESMO Clinical Practice Guidelines for diagnosis, treatment and follow-up. Ann. Oncol. 23(Suppl. 7), vii120-123 (2012).

12. Khan MS, Tsigani T, Rashid M et al. Circulating tumor cells and EpCAM expression in neuroendocrine tumors. Clin. Cancer Res. 17(2), 337-345 (2011).

13. Khan MS, Kirkwood A, Tsigani T et al. Circulating tumor cells as prognostic markers in neuroendocrine tumors. J. Clin. Oncol. 31(3), 365-372 (2013). 
14. Li A, Yu J, Kim H et al. MicroRNA array analysis finds elevated serum miR-1290 accurately distinguishes patients with low-stage pancreatic cancer from healthy and disease controls. Clin. Cancer Res. 19(13), 3600-3610 (2013).

15. Roldo C, Missiaglia E, Hagan JP et al. MicroRNA expression abnormalities in pancreatic endocrine and acinar tumors are associated with distinctive pathologic features and clinical behavior. J. Clin. Oncol. 24(29), 4677-4684 (2006).

16. Childs A, Kirkwood A, Edeline J et al. Ki-67 index and response to chemotherapy in patients with neuroendocrine tumours. Endocr. Relat. Cancer 23(7), 563-570 (2016).

17. Van Essen M, Krenning EP, De Jong M, Valkema R, Kwekkeboom DJ. Peptide receptor radionuclide therapy with radiolabelled somatostatin analogues in patients with somatostatin receptor-positive tumours. Acta Oncol. 46(6), 723-734 (2007).

18. Edfeldt K, Daskalakis K, Backlin C et al. DcR3, TFF3 and Midkine are novel serum biomarkers in small intestinal neuroendocrine tumors. Neuroendocrinology 105(2), 170-181 (2017).

19. Zurita AJ, Khajavi M, Wu HK et al. Circulating cytokines and monocyte subpopulations as biomarkers of outcome and biological activity in sunitinib-treated patients with advanced neuroendocrine tumours. Br. J. Cancer 112(7), 1199-1205 (2015).

20. Modlin IM, Drozdov I, Kidd M. Gut neuroendocrine tumor blood qPCR fingerprint assay: characteristics and reproducibility. Clin. Chem. Lab. Med. 52(3), 419-429 (2014). 